\title{
Epidemiologia dos acidentes ofídicos nos últimos 100 anos no Brasil: uma revisão
}

\author{
Snake bite epidemiology in the last 100 years \\ in Brazil: a review
}

Rosany Bochner 1

Claudio José Struchiner 2

\footnotetext{
1 Centro de Informação Científica e Tecnológica, Fundação Oswaldo Cruz. Av. Brasil 4365, Rio de Janeiro, $R J$ 21045-900, Brasil. rosany@cict.fiocruz.br 2 Programa de Computação Científica, Fundação Oswaldo Cruz. Av. Brasil 4365, Rio de Janeiro, $R J$ 21045-900, Brasil. stru@procc.fiocruz.br
}

\begin{abstract}
We review 30 studies on snake bites in Brazil, published from 1901 to 2000, and conclude that epidemiological analyses conducted in the last 100 years are based on the same variables already identified by Vital Brazil in his pioneering report, i.e., characteristics of the individuals prone to snake bites, the bites themselves, and treatment. The original epidemiological profile was also maintained over the years and indicates that such accidents are more frequent among male farm workers in the 15-49-year age bracket, affecting mainly the lower limbs, and caused by snakes from genus Bothrops.
\end{abstract}

Key words Snake Bites; Accidents; Snakes; Review Literature

Resumo Neste trabalho foram analisados 22 artigos, 4 livros, 3 relatórios e 1 manual publicados no período de 1901 a 2000, que tratam de acidentes ofídicos ocorridos no Brasil. Concluiu-se que as análises epidemiológicas realizadas nos últimos 100 anos são baseadas nas mesmas variáveis já apontadas por Vital Brazil em seu Boletim para Observação de Accidente Ophidico, ou seja, variáveis referentes ao indivíduo, ao evento e ao atendimento. O perfil epidemiológico desses acidentes também se manteve inalterado ao longo dos anos, isto é, são mais comuns em pessoas do sexo masculino, em trabalhadores rurais, na faixa etária de 15 a 49 anos, atingem principalmente os membros inferiores e a maioria desses acidentes é atribuída às serpentes do gênero Bothrops.

Palavras-chave Mordeduras de Cobras; Acidentes; Cobras; Literatura de Revisão 


\section{Introdução}

O primeiro estudo epidemiológico de acidentes ofídicos foi realizado por Vital Brazil em 1901, quando levantou o número de óbitos por picadas de serpentes peçonhentas no Estado de São Paulo, registrando 63, 88 e 104 óbitos em 1897, 1899 e 1900, respectivamente (Brazil, 1901). Em 14 de agosto de 1901, Vital Brazil entregou os primeiros tubos de soros antipeçonhentos para o consumo (Vaz, 1950), e a partir daí passou a distribuir, junto com as ampolas de soro, o Boletim para Observação de Accidente Ophidico, para ser preenchido com dados referentes ao acidente que levou ao uso desse antiveneno. Por meio desse boletim, vários trabalhos foram publicados, como o do próprio Vital Brazil (1911), Penteado (1918), Amaral (1930), Barroso (1944), Fonseca (1949) e Magalhães (1958).

Com o tempo, surgiram outros modelos de ficha para a notificação desses acidentes como os utilizados pelo Estado de São Paulo (Martinez et al., 1995; Ribeiro et al., 1993, 1998), pelos Centros de Informação e Controle de Intoxicações (Carvalho \& Nogueira, 1998; Rodrigues et al., 1988; Torres et al., 1982), pelo Estado do Rio de Janeiro (Gomes et al., 1997), pelo Estado de Roraima (Nascimento, 2000) e pelo Programa Nacional de Ofidismo (Barraviera, 1993; MS/ FUNASA, 1998; MS/SNABS, 1989; Resende et al., 1989).

Com os casos passando a ser atendidos em unidades de saúde, vários estudos foram realizados baseados em prontuários médicos como o de Rosenfeld (1972), Belluomini et al. (1987), Vêncio (1988), Queiroz \& Moritz (1989), Kouyoumdjian et al. (1990), Garcia et al. (1994), Ribeiro et al. (1995), Caiaffa et al. (1997), Borges et al. (1999) e Cardoso (2000).

\section{Objetivo}

Apresentar o perfil epidemiológico dos acidentes ofídicos e as variáveis utilizadas ao longo dos últimos 100 anos nas análises realizadas no país.

\section{Metodologia}

Dada a importância da contribuição de Vital Brazil no campo do ofidismo, primeiramente foi realizada uma análise minuciosa de toda sua produção científica, que se encontra reunida em um único livro, abrangendo desde sua tese de doutoramento, publicada em 1892, até seu último artigo, lançado em 1941 (Pereira Neto, 2002).

Feito isso, partiu-se para uma revisão bibliográfica buscando trabalhos de outros autores sobre acidentes ofídicos ocorridos no Brasil, publicados no período de 1901 a 2000. Foram obtidas 87 referências: 72 artigos, 8 livros, 4 relatórios e 3 manuais. Destas foram selecionadas 30, sendo 22 artigos, 4 livros, 3 relatórios e 1 manual, de acordo com os seguintes critérios: (1) constituir um marco conceitual no tema (Brazil, 1901, 1909, 1911); (2) apresentar dados epidemiológicos de forma detalhada (Amaral, 1930; Barroso, 1944; Belluomini et al., 1987; Cardoso, 2000; Fonseca, 1949; Garcia et al., 1994; Kouyoumdjian et al., 1990; Martinez et al., 1995; Penteado, 1918; Ribeiro et al., 1993, 1998; Rozenfeld, 1972); (3) constituir estudo de um determinado Estado ou do país como um todo (Barraviera, 1993; Borges et al., 1999; Carvalho \& Nogueira, 1998; Gomes et al., 1997; Magalhães, 1958; MS/FUNASA, 1998; MS/SNABS, 1989; Nascimento, 2000; Queiroz \& Moritz, 1989; Resende et al., 1989; Rodrigues et al., 1988; Torres et al., 1982; Vêncio, 1988); (4) apresentar um desenho diferente de estudo (Caiaffa et al., 1997; Ribeiro et al., 1995).

Artigos muito específicos, como os de Ribeiro \& Jorge (1989, 1990), que tratam da epidemiologia e quadro clínico dos acidentes causados por serpentes adultas e filhotes da espécie Bothrops jararaca, assim como o de Barraviera et al. (1989), que avaliaram 40 doentes com diagnóstico de acidente crotálico, não foram selecionados. Artigos apresentados na forma de resumo, formato adotado por alguns periódicos como o Toxicon e a Revista da Sociedade Brasileira de Medicina Tropical, também não foram considerados neste estudo.

Lebrão et al. (1995) analisaram os prontuários médicos de todos os 21 óbitos por acidente ofídico ocorridos no Estado de São Paulo, nos anos de 1988 e 1989, e notificados à Secretaria de Saúde do Estado de São Paulo. Concluíram que $52,4 \%$ dos óbitos não eram previsíveis, que na maioria das vezes a complicação não foi reconhecida, que em três casos não foram tomadas as medidas apropriadas e que em três casos o óbito poderia ter sido evitado. Apesar de se tratar de um artigo interessante, que enfoca a qualidade do atendimento prestado, também não foi incluído em nossa análise pelo fato de ter trabalhado somente com dados de óbito. 


\section{Resultados}

A Tabela 1 apresenta as variáveis utilizadas nas análises realizadas por cada um dos 30 artigos estudados. As variáveis foram reunidas em três grupos, variáveis referentes ao indivíduo, ao evento e ao atendimento.

É interessante verificar que as análises desses acidentes se mantêm em torno das mesmas variáveis apontadas por Vital Brazil em seu Boletim para Observação de Accidente Ophidico, como sexo e idade da vítima, local da picada, espécie e/ou gênero da serpente, tempo decorrido entre o acidente e o atendimento, tipo de soro utilizado, número de ampolas, evolução do caso, avaliação de manifestações clínicas como cegueira, hemorragia, paralisia e inchaço no local da picada, local e data do acidente. Brazil (1914), Amaral (1930) e Magalhães (1958) apresentam o formato desse Boletim.

Ribeiro et al. (1995) entrevistaram 209 dos 322 pacientes acidentados por serpentes peçonhentas e/ou seus acompanhantes e foram os únicos a coletar as seguintes informações: atividade que o paciente desenvolvia no momento do acidente, tipo de vestimenta que usava, eventual presença de anteparos transfixados pelos dentes inoculadores da serpente antes de penetrarem no paciente, observação visual da serpente pelo paciente, comportamento quan-

Tabela 1

Descrição das variáveis utilizadas por cada um dos artigos.

$\begin{array}{llllllllllllll}\text { Artigos } & \end{array}$

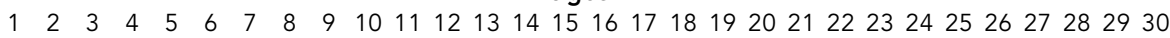

\begin{tabular}{|c|c|c|c|c|c|c|c|c|c|c|c|c|c|c|c|c|c|c|c|c|c|c|c|c|c|c|c|c|}
\hline Indivíduo & & & & & & & & & & & & & & & & & & & & & & & & & & & & \\
\hline Vítima (homem, mulher, criança) & & & $x$ & $x$ & $x$ & $x$ & & & & & & & & & & & & & & & & & & & & & & \\
\hline Sexo & & $x$ & & & & & & $x$ & $x$ & $x$ & $x$ & $x$ & $x$ & $x$ & $x$ & $x$ & $x$ & $x$ & $x$ & $x$ & $X$ & $x$ & $x$ & & $x$ & $x$ & $x$ & \\
\hline Idade & & $x$ & & & & & $x$ & $x$ & $x$ & $x$ & $x$ & $x$ & $x$ & $x$ & $x$ & $x$ & $x$ & $x$ & $x$ & $x$ & $x$ & $x$ & $x$ & & $x$ & $x$ & $x$ & \\
\hline Etnia & & & & & & & & & & & & & & & & & & & & & & $x$ & & & & & & \\
\hline Naturalidade & & & & & & & & & & & & & & & & & & & & & & & & & & $x$ & & \\
\hline Profissão/Ocupação & & & & & & & & & $x$ & & & & & & $x$ & & & $x$ & $x$ & $x$ & & $x$ & & & & $x$ & $x$ & \\
\hline Vestimenta & & & & & & & & & & & & & & & & & & & & $x$ & & & & & & & & \\
\hline Evento & & & & & & & & & & & & & & & & & & & & & & & & & & & & \\
\hline Região geográfica & & & & & & & & & & & & & $x$ & $x$ & & $x$ & & & & & $x$ & & $x$ & & & & & \\
\hline Estado & & & & $x$ & & & & & & & & & $x$ & & & & & & & & & & & & & & & \\
\hline Município & $x$ & & & & & & & & $x$ & $x$ & & & & & $x$ & & & & $x$ & $x$ & & & & & & & $x$ & \\
\hline Zona & & & & & & & & & & & & & & & $x$ & & & & $x$ & & & $x$ & & $x$ & & $x$ & & \\
\hline Local de ocorrência & & & & & & & & & & & & & & & & & & & & & $x$ & & & $x$ & & & & \\
\hline Mês & $x$ & & & & & $x$ & & $x$ & & & & & $x$ & & $x$ & $x$ & $x$ & $x$ & $x$ & $x$ & $x$ & $x$ & $x$ & & & $x$ & $x$ & $x$ \\
\hline Trimestre & & & & $x$ & & & & & & & & & & & & & & & & & & & & & & & & \\
\hline Horário & & & & & & & & & & & & & & & $x$ & & & & & $x$ & & $x$ & & $x$ & & $x$ & $x$ & \\
\hline Circunstância & & & & & & & & & $x$ & & & & & & $x$ & & & & & $x$ & & $x$ & & & & & & \\
\hline Local da picada & & $x$ & $x$ & $x$ & $x$ & & & $x$ & $x$ & $x$ & $x$ & $x$ & $x$ & $x$ & $x$ & $x$ & $x$ & $x$ & & $x$ & $x$ & $x$ & $x$ & & $x$ & $x$ & & $x$ \\
\hline Animal peçonhento envolvido & & & & & & & $x$ & $x$ & $x$ & & & & & & & & & & & & $x$ & & & & & & & \\
\hline Identificação do animal & & & & & & & & & & & & $x$ & & & $x$ & & & & & & & & & & & & & \\
\hline Gênero da serpente & & $x$ & $x$ & $x$ & $x$ & $x$ & $x$ & $x$ & $x$ & $x$ & $x$ & $x$ & $x$ & $x$ & $x$ & $x$ & $x$ & $x$ & & $x$ & & $x$ & $x$ & $x$ & $x$ & $x$ & $x$ & $x$ \\
\hline Espécie da serpente & & $x$ & $x$ & & $x$ & $x$ & & & & $x$ & & $x$ & & & $x$ & & & & & $x$ & & & & $x$ & & & & $x$ \\
\hline Grau de maturidade da serpente & & & & & & & & & & & & & & & & & & & & $x$ & & & & & & & & \\
\hline Sexo da serpente & & & & & & & & & & & & & & & & & & & & $x$ & & & & & & & & \\
\hline Observação visual da serpente & & & & & & & & & & & & & & & & & & & & $x$ & & & & & & & & \\
\hline Comportamento da serpente & & & & & & & & & & & & & & & & & & & & $x$ & & & & & & & & \\
\hline Utilização do substrato & & & & & & & & & & & & & & & & & & & & & & & & $x$ & & & & \\
\hline Período de atividade da serpente & & & & & & & & & & & & & & & & & & & & & & & & $x$ & & & & \\
\hline Dieta da serpente & & & & & & & & & & & & & & & & & & & & & & & & $x$ & & & & \\
\hline
\end{tabular}

(continua) 
Tabela 1 (continuação)

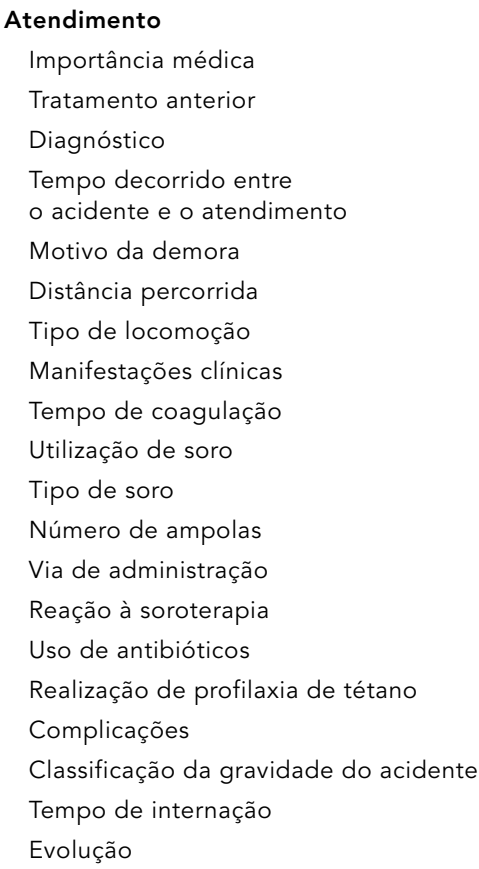

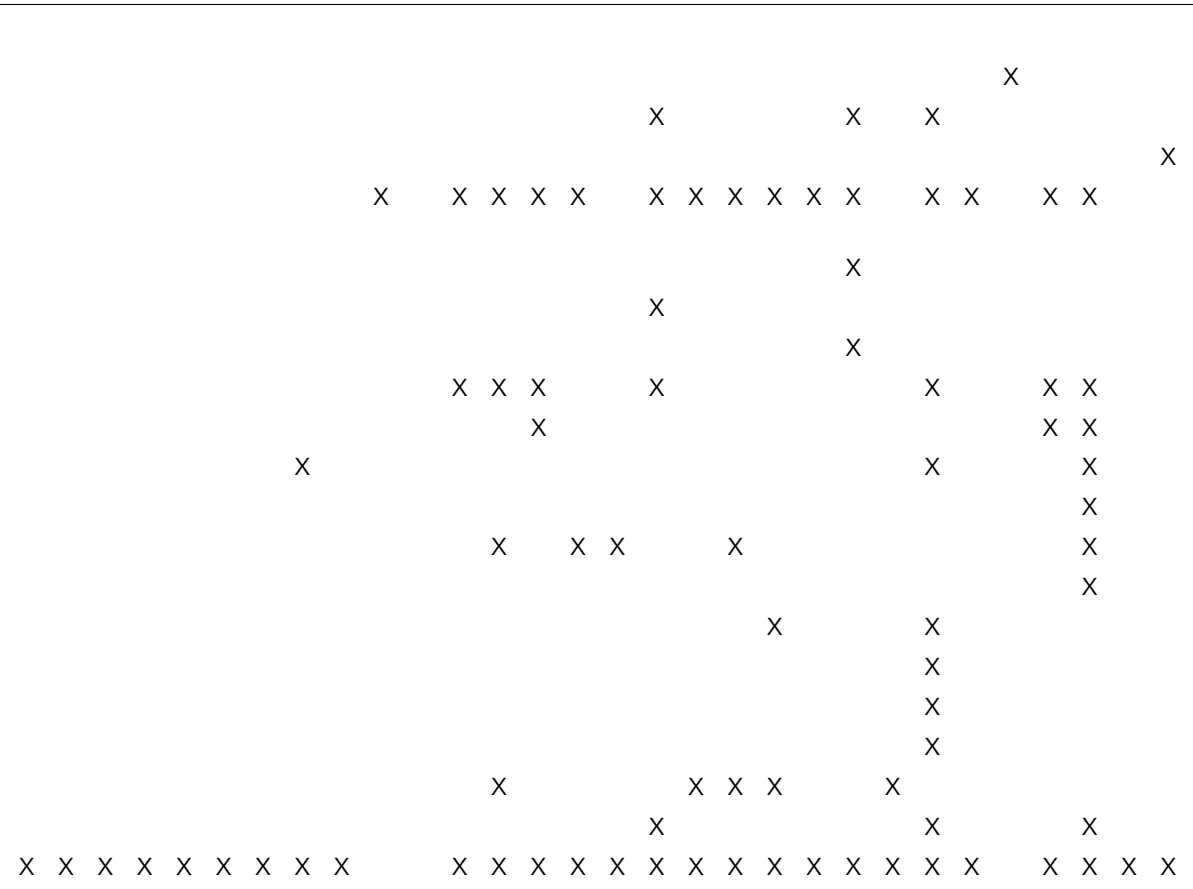

Artigos: 1 - Brazil (1901); 2 - Brazil (1909); 3 - Brazil (1911); 4 - Penteado (1918); 5 - Amaral (1930); 6 - Barroso (1944);

7 - Fonseca (1949); 8 - Magalhães (1958); 9 - Rosenfeld (1972); 10 - Torres \& Carlotto (1982); 11 - Belluomini et al. (1987);

12 - Vêncio (1988); 13 - Rodrigues et al. (1988); 14 - Queiroz \& Moritz (1989); 15 - Resende et al. (1989); 16 - MS/SNABS (1989);

17 - Kouyoumdjian et al. (1990); 18 - Barraviera (1993); 19 - Ribeiro et al. (1993); 20 - Garcia et al. (1994); 21 - Martinez et al.

(1995); 22 - Ribeiro et al. (1995); 23 - Gomes et al.(1997); 24 - Caiaffa et al. (1997); 25 - MS/FUNASA (1998); 26 - Carvalho

\& Nogueira (1998); 27 - Ribeiro et al. (1998); 28 - Borges et al. (1999); 29 - Nascimento (2000); 30 - Cardoso (2000).

to aos movimentos da serpente no momento da picada e após, explicitação do motivo de eventual demora na busca de atendimento e dos meios de locomoção utilizados no transporte do paciente.

Caiaffa et al. (1997) realizaram um estudo clínico-epidemiológico durante um período de sete anos em um hospital de emergências de Belo Horizonte, comparando 310 pacientes hospitalizados por acidentes ofídicos (casos) com 310 pacientes hospitalizados por outras causas (controles), pareados por idade e sexo. Comparando os casos e controles, concluíram que aqueles de origem rural, ou relatando atividade agrícola, apresentaram riscos de acidentes ofídicos, estimado pela odds relativa (OR), de 14,7 e 6,7 , respectivamente, sendo portanto identificados como um grupo de alto risco. Com relação ao tratamento, os casos mostraram um risco de reações anafiláticas precoces 13,5 vezes maior quando comparados aos respectivos controles, e este risco sobe para 30,3 no grupo etário de $\geq 20$ anos. Os casos apresentaram também um maior risco de pirexia $(\mathrm{OR}=11,7)$, mostrando uma associação mais acentuada no grupo etário de menores de 19 anos (OR = 16,6). Os casos também diferem dos controles, considerando alguns procedimentos durante a hospitalização, como por exemplo, os casos tiveram um menor tempo de hospitalização, receberam antibióticos $(24,2 \%)$ e profilaxia de tétano $(42,9 \%)$ em menores proporções do que os controles, no entanto, casos e controles apresentaram a mesma proporção de terapia intravenosa $(99,0 \%)$. Este artigo foi o único a considerar a variável etnia, concluindo que $79,6 \%$ dos acidentes ocorreram em pessoas não brancas.

Com exceção dos artigos de Caiaffa et al. (1997) e de Ribeiro et al. (1995), os demais se limitaram a realizar estudos puramente descritivos, diferindo apenas no número de variáveis analisadas.

Além das estatísticas sobre os acidentes, Amaral (1930) e Fonseca (1949), baseados no Instituto Butantan, e Magalhães (1958), baseado no Instituto Ezequiel Dias, dão ênfase aos dados referentes ao recebimento de serpentes, produção de soro e distribuição geográfica das cobras. 
Carvalho \& Nogueira (1998) analisaram além dos dados referentes aos acidentes ofídicos, 213 serpentes oriundas da área urbana de Cuiabá, doados por populares ao Núcleo de Ofiologia Regional de Mato Grosso (NORMAT) e aqueles coligidos por Christine Strüssmann, depositados na coleção de vertebrados do Instituto de Biociências da Universidade Federal de Mato Grosso, durante o período de 1986 a 1993. Para cada espécie foram compilados os aspectos ecológicos relacionados, isto é, utilização do substrato $(71,5 \%$ de espécies terrestres, cerca de $14 \%$ de semi-arbóreas, $11 \%$ de aquáticas e semi-aquáticas, $3 \%$ de fossórias), período de atividade (57\% são diurnas, $20 \%$ noturnas, $20 \%$ diurnas/noturnas) e dieta (cerca de $25 \%$ do número total das espécies alimentam-se especialmente de anfíbios e/ou lagartos, 18\% consomem, além disso, pequenos mamíferos).

Penteado (1918), Amaral (1930), Barroso (1944), Fonseca (1949) e Magalhães (1958) usaram a variável vítima, que contava com as categorias homem, mulher, criança e animal, de forma a substituir as variáveis sexo e idade.

As variáveis de atendimento passam a ser mais comuns nos artigos mais recentes, a saber, naqueles publicados a partir de 1988. É importante lembrar que, no início, a aplicação do soro era feita por pessoas leigas sem acompanhamento médico, pois uma das estratégias adotadas por Vital Brazil para difundir a utilização do soro foi a substituição do sistema de compra de cobras venenosas, instituído por Lutz, pelo de permuta de ofídios com ampolas de soro antipeçonhento (Silva Jr., 1956).

Brazil (1911) relata 50 casos tratados pelo soro antiofídico, considerados por ele dignos de atenção. Também afirma que todas as vítimas de acidentes ofídicos que procuram tratamento específico em tempo oportuno têm sido salvas e que a estatística do Estado de São Paulo já acusa notável decréscimo no número de óbitos.

Penteado (1918) analisou 1.393 casos tratados com soro, destes, 1.358 tiveram um bom resultado e 25 vieram a óbito. Analisando estes óbitos, concluiu que o fracasso do tratamento não foi devido ao soro, mas a causas diversas como o atraso no atendimento e a aplicação de quantidades insuficientes de soro.

Magalhães (1958) apresenta o relato de 162 casos extraídos do arquivo de 980 registros do Instituto Biológico Ezequiel Dias.

A Tabela 2 apresenta um diagrama ramo-folha (Tukey, 1977), tendo como ramo os anos compreendidos no período de 1901 a 2000 e as folhas representando os estudos realizados com base em dados referentes àquele ano, indican- do a origem geográfica dos dados pela sigla dos Estados ou por BR para os dados nacionais. Os artigos correspondentes também são indicados entre parênteses. Por exemplo, o artigo 4 referente a Penteado (1918), que analisou dados de São Paulo compreendidos no período de 1902 a 1916, irá gerar 15 “folhas”, uma para cada ano estudado, com a seguinte notação SP(4). O diagrama é apresentado em dois períodos, de 1901 a 1953 e de 1954 a 2000. Para melhor visualização da distribuição de trabalhos ao longo dos anos, gire a tabela em $90^{\circ}$ no sentido anti-horário. Podemos observar que nenhum dos artigos selecionados se debruçou sobre os dados dos anos de 1901, de 1946 a 1953 e de 1999 a 2000 . Uma grande concentração de artigos é observada no período de 1986 a 1993. Podemos notar que até 1945 apenas os Estados de São Paulo, Rio de Janeiro e Minas Gerais realizaram estudos, o que pode ser explicado pela localização dos Institutos Butantan em São Paulo (criado em 1900), Vital Brazil no Rio de Janeiro (criado em 1919) e Ezequiel Dias em Minas Gerais (criado em 1907, e o posto antiofídico em 1918).

A Tabela 3 apresenta a origem geográfica dos dados tratados pelos artigos. O grande número de trabalhos acerca do Estado de São Paulo (50\%) já era esperado, uma vez que a história do ofidismo está intimamente ligada a esse Estado por diversos fatores, como a descoberta do soro antiofídico por Vital Brazil e a criação do Instituto Butantan. Além disso, o Estado de São Paulo foi pioneiro na criação da vigilância epidemiológica desse tipo de agravo à saúde, ainda no segundo semestre de 1981. Dessa forma, os acidentes por animais peçonhentos que exigissem a utilização de soro deveriam ser, obrigatoriamente, notificados à Secretaria de Saúde de São Paulo por meio de uma ficha denominada Ficha de Registro de Aplicação de Soro (FRAS). Assim, a reposição dos estoques de soro nas unidades de saúde ficava dependente da notificação dos acidentes e das quantidades de soro utilizadas. Em 1983, foi instituído um novo modelo de FRAS, que procurava recuperar informações de natureza clínica dos atendimentos (Cardoso, 1993). Estas informações são ainda mais surpreendentes ao pensarmos que o Programa Nacional de Ofidismo só surgiu em junho de 1986, fato que justifica o número reduzido de trabalhos de cunho nacional (13,3\%).

A Tabela 4 apresenta o perfil epidemiológico dos acidentes ofídicos segundo as principais variáveis utilizadas pelos 30 artigos estudados. Podemos verificar que as conclusões apresentadas nesta tabela estão em consonância com 
Tabela 2

Diagrama ramo-folha, freqüência de estudos para cada ano, origem geográfica dos dados e indicação do artigo correspondente entre parênteses.

\begin{tabular}{|c|c|c|c|c|c|c|c|c|c|c|c|c|c|}
\hline 1901 & & & & & 1954 & $\mathrm{SP}(9)$ & & & & & & & \\
\hline 1902 & $\mathrm{SP}(4)$ & $\mathrm{SP}(5)$ & $\mathrm{SP}(7)$ & & 1955 & $\mathrm{SP}(9)$ & & & & & & & \\
\hline 1903 & $\mathrm{SP}(4)$ & $\mathrm{SP}(5)$ & $\mathrm{SP}(7)$ & & 1956 & $\mathrm{SP}(9)$ & & & & & & & \\
\hline 1904 & $\mathrm{SP}(4)$ & $\mathrm{SP}(5)$ & $\mathrm{SP}(7)$ & & 1957 & $\mathrm{SP}(9)$ & & & & & & & \\
\hline 1905 & $\mathrm{SP}(4)$ & $\mathrm{SP}(5)$ & $\mathrm{SP}(7)$ & & 1958 & $\mathrm{SP}(9)$ & & & & & & & \\
\hline 1906 & $\mathrm{SP}(4)$ & $\mathrm{SP}(5)$ & $\mathrm{SP}(7)$ & & 1959 & $\mathrm{SP}(9)$ & & & & & & & \\
\hline 1907 & $\mathrm{SP}(4)$ & $\mathrm{SP}(5)$ & $\mathrm{SP}(7)$ & & 1960 & $\mathrm{SP}(9)$ & & & & & & & \\
\hline 1908 & $\mathrm{SP}(4)$ & $\mathrm{SP}(5)$ & $\mathrm{SP}(7)$ & & 1961 & $\mathrm{SP}(9)$ & & & & & & & \\
\hline 1909 & $\mathrm{SP}(4)$ & $\mathrm{SP}(5)$ & $\mathrm{SP}(7)$ & & 1962 & $\mathrm{SP}(9)$ & & & & & & & \\
\hline 1910 & $\mathrm{SP}(4)$ & $\mathrm{SP}(5)$ & $\mathrm{SP}(7)$ & & 1963 & $\mathrm{SP}(9)$ & & & & & & & \\
\hline 1911 & $\mathrm{SP}(4)$ & $\mathrm{SP}(5)$ & $\mathrm{SP}(7)$ & & 1964 & $\mathrm{SP}(9)$ & & & & & & & \\
\hline 1912 & $\mathrm{SP}(4)$ & $\mathrm{SP}(5)$ & $\mathrm{SP}(7)$ & & 1965 & $\mathrm{SP}(9)$ & & & & & & & \\
\hline 1913 & $\mathrm{SP}(4)$ & $\mathrm{SP}(5)$ & $\mathrm{SP}(7)$ & & 1966 & $\mathrm{SP}(30)$ & & & & & & & \\
\hline 1914 & $\mathrm{SP}(4)$ & $\mathrm{SP}(5)$ & $\mathrm{SP}(7)$ & & 1967 & $S P(30)$ & & & & & & & \\
\hline 1915 & $\mathrm{SP}(4)$ & $\mathrm{SP}(5)$ & $\mathrm{SP}(7)$ & & 1968 & $\mathrm{SP}(30)$ & & & & & & & \\
\hline 1916 & $\mathrm{SP}(4)$ & $\mathrm{SP}(5)$ & $\mathrm{SP}(7)$ & & 1969 & $S P(30)$ & & & & & & & \\
\hline 1917 & $\mathrm{SP}(5)$ & $\mathrm{SP}(7)$ & & & 1970 & $\mathrm{SP}(30)$ & & & & & & & \\
\hline 1918 & $\mathrm{SP}(5)$ & $\mathrm{SP}(7)$ & $M G(8)$ & & 1971 & $\mathrm{SP}(30)$ & & & & & & & \\
\hline 1919 & $\mathrm{SP}(5)$ & $\mathrm{RJ}(6)$ & $\mathrm{SP}(7)$ & $M G(8)$ & 1972 & $S P(30)$ & & & & & & & \\
\hline 1920 & $\mathrm{SP}(5)$ & $\mathrm{RJ}(6)$ & $\mathrm{SP}(7)$ & $M G(8)$ & 1973 & $\mathrm{GO}(12)$ & $\mathrm{SP}(30)$ & & & & & & \\
\hline 1921 & $\mathrm{SP}(5)$ & $\mathrm{RJ}(6)$ & $\mathrm{SP}(7)$ & $M G(8)$ & 1974 & $\mathrm{GO}(12)$ & $\mathrm{SP}(30)$ & & & & & & \\
\hline 1922 & $\mathrm{SP}(5)$ & $\mathrm{RJ}(6)$ & $\mathrm{SP}(7)$ & $M G(8)$ & 1975 & $\mathrm{GO}(12)$ & $\mathrm{SP}(30)$ & & & & & & \\
\hline 1923 & $\mathrm{SP}(5)$ & $\mathrm{RJ}(6)$ & $\mathrm{SP}(7)$ & $M G(8)$ & 1976 & $\mathrm{GO}(12)$ & $\mathrm{SP}(30)$ & & & & & & \\
\hline 1924 & $\mathrm{SP}(5)$ & $\mathrm{RJ}(6)$ & $\mathrm{SP}(7)$ & $M G(8)$ & 1977 & $\mathrm{RS}(10)$ & $\mathrm{GO}(12)$ & $\mathrm{SP}(30)$ & & & & & \\
\hline 1925 & $\mathrm{SP}(5)$ & $\mathrm{RJ}(6)$ & $\mathrm{SP}(7)$ & $M G(8)$ & 1978 & $\mathrm{RS}(10)$ & $\mathrm{GO}(12)$ & & & & & & \\
\hline 1926 & $\mathrm{SP}(5)$ & $\mathrm{RJ}(6)$ & $\mathrm{SP}(7)$ & $M G(8)$ & 1979 & $\mathrm{RS}(10)$ & $\mathrm{GO}(12)$ & & & & & & \\
\hline 1927 & $\mathrm{SP}(5)$ & $\mathrm{RJ}(6)$ & $\mathrm{SP}(7)$ & $M G(8)$ & 1980 & $\mathrm{RS}(10)$ & $M G(24)$ & & & & & & \\
\hline 1928 & $\mathrm{SP}(5)$ & $\mathrm{RJ}(6)$ & $\mathrm{SP}(7)$ & $M G(8)$ & 1981 & $\mathrm{RS}(10)$ & $S P(17)$ & $M G(24)$ & & & & & \\
\hline 1929 & $\mathrm{SP}(5)$ & $\mathrm{RJ}(6)$ & $\mathrm{SP}(7)$ & $M G(8)$ & 1982 & $\mathrm{SP}(17)$ & $M G(24)$ & & & & & & \\
\hline 1930 & $\mathrm{RJ}(6)$ & $\mathrm{SP}(7)$ & $M G(8)$ & & 1983 & $\mathrm{SP}(11)$ & SP(17) & $M G(24)$ & & & & & \\
\hline 1931 & $\mathrm{RJ}(6)$ & $\mathrm{SP}(7)$ & $\mathrm{MG}(8)$ & & 1984 & $\mathrm{SP}(17)$ & MG(24) & & & & & & \\
\hline 1932 & $\mathrm{RJ}(6)$ & $\mathrm{SP}(7)$ & $M G(8)$ & & 1985 & SC(14) & $\mathrm{SP}(17)$ & $\mathrm{SP}(21)$ & MG(24) & & & & \\
\hline 1933 & $\mathrm{RJ}(6)$ & $\mathrm{SP}(7)$ & $M G(8)$ & & 1986 & $\mathrm{BA}(13)$ & SC(14) & $\mathrm{BR}(15)$ & $\mathrm{SP}(17)$ & $\mathrm{SP}(19)$ & $\mathrm{SP}(21)$ & $M G(24)$ & \\
\hline 1934 & $\mathrm{RJ}(6)$ & $\mathrm{SP}(7)$ & $M G(8)$ & & 1987 & $\mathrm{SC}(14)$ & $\mathrm{BR}(15)$ & $\mathrm{SP}(17)$ & $\mathrm{BR}(18)$ & $\mathrm{SP}(19)$ & $\mathrm{SP}(20)$ & $\mathrm{SP}(21)$ & \\
\hline 1935 & $\mathrm{RJ}(6)$ & $\mathrm{SP}(7)$ & MG(8) & & 1988 & $\mathrm{BR}(18)$ & $\mathrm{SP}(19)$ & $\mathrm{SP}(20)$ & $\mathrm{SP}(21)$ & $\mathrm{SP}(22)$ & $\mathrm{MS}(26)$ & $\mathrm{SP}(27)$ & \\
\hline 1936 & $\mathrm{RJ}(6)$ & $\mathrm{SP}(7)$ & $M G(8)$ & & 1989 & $\mathrm{BR}(16)$ & $\mathrm{BR}(18)$ & $\mathrm{SP}(19)$ & $\mathrm{SP}(20)$ & $\mathrm{SP}(21)$ & $\mathrm{MS}(26)$ & $\mathrm{SP}(27)$ & $\operatorname{AM}(28)$ \\
\hline 1937 & $\mathrm{RJ}(6)$ & $\mathrm{SP}(7)$ & $M G(8)$ & & 1990 & $\mathrm{SP}(20)$ & RJ(23) & $\mathrm{BR}(25)$ & $\mathrm{MS}(26)$ & $\mathrm{SP}(27)$ & $\mathrm{AM}(28)$ & & \\
\hline 1938 & $\mathrm{RJ}(6)$ & $\mathrm{SP}(7)$ & MG(8) & & 1991 & $S P(20)$ & RJ(23) & $\mathrm{BR}(25)$ & $\mathrm{MS}(26)$ & $\mathrm{SP}(27)$ & $\mathrm{AM}(28)$ & & \\
\hline 1939 & $\mathrm{RJ}(6)$ & $\mathrm{SP}(7)$ & $M G(8)$ & & 1992 & $S P(20)$ & RJ(23) & $\mathrm{BR}(25)$ & $\mathrm{MS}(26)$ & $\mathrm{SP}(27)$ & $\mathrm{AM}(28)$ & $\mathrm{RR}(29)$ & \\
\hline 1940 & $\mathrm{RJ}(6)$ & $\mathrm{SP}(7)$ & $M G(8)$ & & 1993 & RJ(23) & $\mathrm{BR}(25)$ & $\mathrm{MS}(26)$ & $\mathrm{SP}(27)$ & $\mathrm{AM}(28)$ & $\mathrm{RR}(29)$ & & \\
\hline 1941 & $\mathrm{RJ}(6)$ & $\mathrm{SP}(7)$ & & & 1994 & RJ(23) & $\mathrm{AM}(28)$ & $\mathrm{RR}(29)$ & & & & & \\
\hline 1942 & $\mathrm{RJ}(6)$ & $\mathrm{SP}(7)$ & & & 1995 & RJ(23) & $\mathrm{AM}(28)$ & $\operatorname{RR}(29)$ & & & & & \\
\hline 1943 & $\mathrm{SP}(7)$ & & & & 1996 & RJ(23) & $\mathrm{AM}(28)$ & RR(29) & & & & & \\
\hline 1944 & $\mathrm{SP}(7)$ & & & & 1997 & $\mathrm{RR}(29)$ & & & & & & & \\
\hline 1945 & $\mathrm{SP}(7)$ & & & & 1998 & $\mathrm{RR}(29)$ & & & & & & & \\
\hline 1946 & & & & & 1999 & & & & & & & & \\
\hline 1947 & & & & & 2000 & & & & & & & & \\
\hline \multicolumn{14}{|l|}{1948} \\
\hline \multicolumn{14}{|l|}{1949} \\
\hline \multicolumn{14}{|l|}{1950} \\
\hline \multicolumn{14}{|l|}{1951} \\
\hline \multicolumn{14}{|l|}{1952} \\
\hline 1953 & & & & & & & & & & & & & \\
\hline
\end{tabular}

Legenda: (4) Penteado (1918); (5) Amaral (1930); (6) Barroso (1944); (7) Fonseca (1949); (8) Magalhães (1958); (9) Rosenfeld (1972);

(10) Torres \& Carlotto (1982); (11) Belluomini et al. (1987); (12) Vêncio (1988); (13) Rodrigues et al. (1988); (14) Queiroz \& Moritz (1989);

(15) Resende et al. (1989); (16) MS/SNABS (1989); (17) Kouyoumdjian et al. (1990); (18) Barraviera (1993); (19) Ribeiro et al. (1993);

(20) Garcia et al. (1994); (21) Martinez et al. (1995); (22) Ribeiro et al. (1995); (23) Gomes et al.(1997); (24) Caiaffa et al. (1997);

(25) MS/FUNASA (1998); (26) Carvalho \& Nogueira (1998); (27) Ribeiro et al. (1998); (28) Borges et al. (1999); (29) Nascimento (2000);

(30) Cardoso (2000). 
o seguinte perfil apresentado por Vital Brazil em seu livro datado de 1911:

- A jararaca é a espécie que determina a maior parte dos acidentes, fato que se harmoniza com a abundância em que é encontrada e com a sua distribuição geográfica na região Sul Americana;

- Os homens são vitimados com maior freqüência do que as mulheres, o que se explica perfeitamente pelo tipo de trabalho adotado geralmente por um e por outro sexo;

- Os indivíduos maiores de 15 anos são vitimados com maior freqüência;

- Os membros inferiores são os mais atingidos em cerca de $75 \%$ dos casos.

\section{Discussão}

Benchimol \& Teixeira (1993) e Pereira Neto (2000) salientam o esforço de Vital Brazil em conquistar a aceitação e o reconhecimento junto à comunidade científica e ao Estado, e também em convencer a sociedade da importância e eficácia do soro. Nesse sentido, a produção e divulgação de dados sobre os acidentes ofídicos mereceram atenção especial de Vital Brazil. No entanto, seus dois trabalhos publicados em 1901 e 1909 tratam apenas dos óbitos registrados no Estado de São Paulo nos anos de 1897, 1899 e 1900. Seu livro publicado em 1911 constitui o primeiro trabalho epidemiológico baseado em casos; contudo, os dados de que lança mão em sua análise não são apresentados de forma detalhada e nem mesmo o período de referência é informado.

Vital Brazil (1911) e Magalhães (1918) serviram-se do relato de casos como forma de mostrar a eficácia do tratamento por soro.

Neste estudo foram observados apenas quatro trabalhos de cunho nacional, dois publicados em 1989, um em 1993 e o último em 1998, sendo republicado em 2001. Mais preocupante que o número reduzido de estudos nacionais é o fato de o Ministério da Saúde (MS) publicar um trabalho em 1998, republicá-lo em 2001 (MS/FUNASA, 2001), com dados referentes aos anos de 1990 a 1993, deixando claro que hoje vivemos num período de carência de informações, fruto da pouca importância política que vem sendo dada a esse tipo de agravo à saúde.

O período de 1986 a 1993 apresenta a maior concentração de estudos, o que pode ser expressado pelo bom funcionamento do sistema de informação concernente aos acidentes por animais peçonhentos nesse período, graças ao condicionamento da distribuição do soro com as notificações dos casos (Bochner \& Struchiner, 2002).

\begin{tabular}{|c|c|c|}
\hline \multicolumn{3}{|c|}{$\begin{array}{l}\text { Distribuição dos } 30 \text { trabalhos analisados de acordo } \\
\text { com a origem de seus dados. }\end{array}$} \\
\hline Região/Estado & $\mathbf{n}$ & $\%$ \\
\hline Norte & 2 & 6,6 \\
\hline Amazonas & 1 & 3,3 \\
\hline Roraima & 1 & 3,3 \\
\hline Nordeste & 1 & 3,3 \\
\hline Bahia & 1 & 3,3 \\
\hline Sudeste & 19 & 63,4 \\
\hline São Paulo & 15 & 50,0 \\
\hline Rio de Janeiro & 2 & 6,7 \\
\hline Minas Gerais & 2 & 6,7 \\
\hline Sul & 2 & 6,6 \\
\hline Santa Catarina & 1 & 3,3 \\
\hline Rio Grande do Sul & 1 & 3,3 \\
\hline Centro-Oeste & 2 & 6,6 \\
\hline Mato Grosso do Sul & 1 & 3,3 \\
\hline Goiás & 1 & 3,3 \\
\hline Brasil & 4 & 13,3 \\
\hline Total & 30 & 100,0 \\
\hline
\end{tabular}

Ao buscarmos uma explicação para a inexistência de trabalhos debruçados sobre os dados referentes ao período de 1946 a 1953, deparamos com os seguintes fatos: o último trabalho de Vital Brazil data de 1941; Vital Brazil faleceu em 1950 e as três revistas científicas especializadas que editou, Archivos do Instituto Vital Brazil (1923/1927), Boletim do Instituto Vital Brazil (1927/1945) e Biologia Médica (1934/1939 e 1942/1946), encerraram suas atividades antes de 1946 (Pereira Neto, 2000).

\section{Conclusão}

As variáveis que estão presentes em mais de $50 \%$ dos estudos, como sexo, idade, mês de ocorrência, local da picada, gênero da serpente, tempo decorrido entre o acidente e o atendimento e evolução, já se encontravam presentes no Boletim para Observação de Accidente Ophidico criado por Vital Brazil ainda em 1901.

Ribeiro et al. (1995) e Caiaffa et al. (1997) poderiam, segundo as metodologias utilizadas em seus trabalhos, ter explorado outras variáveis que pudessem ser utilizadas para a defini- 
Tabela 4

Perfil epidemiológico dos acidentes ofídicos nos últimos 100 anos por intermédio das principais variáveis utilizadas pelos 30 trabalhos.

\begin{tabular}{|c|c|}
\hline Variáveis & Perfil epidemiológico \\
\hline \multicolumn{2}{|l|}{ Indivíduo } \\
\hline Vítima & Homem \\
\hline Sexo & Masculino \\
\hline Idade & 15 a 49 anos \\
\hline Profissão/Ocupação & Lavrador \\
\hline \multicolumn{2}{|l|}{ Evento } \\
\hline Zona & Rural \\
\hline Local de ocorrência & Campo \\
\hline Mês do acidente & Novembro a abril \\
\hline Trimestre do acidente & 1 의 e 4 으 \\
\hline Horário do acidente & Diurno \\
\hline Circunstância do acidente & Trabalho \\
\hline Local da picada & Membros inferiores \\
\hline Animal peçonhento & Serpente \\
\hline Gênero & Bothrops \\
\hline Espécie & Bothrops jararaca \\
\hline \multicolumn{2}{|l|}{ Atendimento } \\
\hline Tratamento anterior & Garroteamento \\
\hline $\begin{array}{l}\text { Tempo decorrido entre o acidente } \\
\text { e o atendimento }\end{array}$ & Menos de 6 horas \\
\hline \multirow[t]{2}{*}{ Manifestações clínicas } & Locais: dor, edema \\
\hline & $\begin{array}{l}\text { Sistêmicas: insuficiência renal, } \\
\text { hemorragia }\end{array}$ \\
\hline Utilização de soro & Em mais da metade dos casos \\
\hline Tipo de soro & Antibotrópico \\
\hline Número de ampolas & Média de 6 ampolas/caso \\
\hline Via de administração & Endovenosa \\
\hline Reação à soroterapia & Ausente na maioria dos casos \\
\hline Classificação da gravidade do acidente & Moderado \\
\hline Tempo de internação & 1 a 5 dias \\
\hline Evolução & $\begin{array}{l}\text { Letalidade geral de 0,45\% } \\
\text { (Bothrops: 0,31\%; Crotalus: } \\
\text { 1,87\%; Lachesis: 0,95\%; } \\
\text { Micrurus: 0,52\%) }\end{array}$ \\
\hline
\end{tabular}

ção de medidas de prevenção e controle desses acidentes, bem como para explicar o porquê de um município apresentar mais ou menos casos que seus vizinhos, ou mesmo para elucidar o fato de possuir ou não certas espécies de serpentes. Assim, é recomendado analisar a relação entre variáveis ambientais e sócio-econômicas na incidência desse tipo de acidente, bem como estudar os hábitos desses animais.

A epidemiologia dos acidentes ofídicos aponta para um perfil que se mantém inalterado ao longo dos últimos 100 anos no Brasil, vale dizer, ocorrem com maior freqüência no início e no final do ano, em pessoas do sexo masculino, em trabalhadores rurais, na faixa etária produtiva de 15 a 49 anos; atingem sobretudo os membros inferiores; e a maioria desses acidentes é atribuída ao gênero Bothrops. Segundo Azevedo-Marques et al. (1992), 85\% das notificações de acidentes ofídicos feitas ao MS referem-se ao gênero Bothrops e somente $10 \%$ ao gênero Crotalus; para as regiões norte e centro do estado de São Paulo, entretanto, o percentual de acidentes crotálicos é de $20 \%$, o maior do país.

Vital Brazil é tão atual que, em 1909, baseado apenas nos óbitos registrados pelo Estado de São Paulo e em uma letalidade de $25 \%$, estimou em 19.200 o número de acidentes ofídicos para todo o Brasil, número este muito próximo dos cerca de 20 mil acidentes ofídicos notificados anualmente no país (MS/FUNASA/CENEPI, 1999). Além disso, em pleno século XXI teve toda sua produção científica reunida em um único livro (Pereira Neto, 2002). Este projeto, pensado ainda em 1950 pelos Anais Paulistas de Medicina e Cirurgia como uma forma de homenageá-lo após sua morte, foi erroneamente abandonado, pois acreditaram em uma perda de atualidade em sua obra que jamais ocorreu (Ribeiro, 1950). 


\section{Referências}

AMARAL, A., 1930. Campanhas anti-ophidicas. $M e$ mórias do Instituto Butantan, 5:195-232.

AZEVEDO-MARQUES, M. M.; CUPO, P. \& HERING, S. E., 1992. Acidentes por animais peçonhentos. $\mathrm{Me}$ dicina, Ribeirão Preto, 25:539-554.

BARRAVIERA, B., 1993. Estudo clínico dos acidentes ofídicos. Jornal Brasileiro de Medicina, 65:209250.

BARRAVIERA, B.; BONJORNO Jr., J. C.; ARAKAKI, D.; DOMINGUES, M. A. C.; PEREIRA, P. C. M.; MENDES, R. P.; MACHADO, J. M. \& MEIRA, D. A., 1989. A retrospective study of 40 victims of Crotalus snake bites. Analysis of the hepatic necrosis observed in one patient. Revista da Sociedade Brasileira de Medicina Tropical, 22:5-12.

BARROSO, R. D., 1944. Ofidismo no Brasil. Boletim do Instituto Vital Brazil, 26:35-47.

BELLUOMINI, H. E.; WAKAMATSU, C. T.; LUCAS, S. M. \& CARDOSO, J. L. C., 1987. Acidentes do trabalho por animais peçonhentos. Revista Brasileira de Saúde Ocupacional, 15:38-42.

BENCHIMOL, J. L. \& TEIXEIRA, L. A., 1993. Cobras, Lagartos \& Outros Bichos: Uma História Comparada dos Institutos Oswaldo Cruz e Butantan. Rio de Janeiro: Editora UFRJ.

BOCHNER, R. \& STRUCHINER, C. J., 2002. Acidentes por animais peçonhentos e sistemas nacionais de informação. Cadernos de Saúde Pública, 18:735746.

BORGES, C. C.; SADAHIRO, M. \& SANTOS, M. C., 1999. Aspectos epidemiológicos e clínicos dos acidentes ofídicos ocorridos nos municípios do Estado do Amazonas. Revista da Sociedade Brasileira de Medicina Tropical, 32:637-646.

BRAZIL, V., 1901. Contribuição ao estudo do veneno ophidico. Revista Médica de São Paulo, IV:255260.

BRAZIL, V., 1909. As cobras venenosas e o tratamento específico do ofidismo. Imprensa Médica, XVII:1721.

BRAZIL, V., 1911. A Defesa Contra o Ophidismo. São Paulo: Pocai \& Weiss.

BRAZIL, V., 1914. La Défense Contre l'Ophidisme. 2eme Ed. São Paulo: Pocai \& Weiss.

CAIAFFA, W. T.; ANTUNES, C. M. F.; OLIVEIRA, H. R. \& DINIZ, C. R., 1997. Epidemiological and clinical aspects of snakebite in Belo Horizonte, Southeast Brazil. Revista do Instituto de Medicina Tropical de São Paulo, 39:113-118.

CARDOSO, J. L. C., 1993. Acidentes por Animais Peçonhentos na Coordenação de Zoonoses e Animais Peçonhentos - Comentários e Sugestões. Brasília: Ministério da Saúde. (mimeo.)

CARDOSO, J. L. C., 2000. Ofidismo. Aracneísmo. Escorpionismo. Epidemiologia. Patogenia e clínica. Diagnóstico e terapêutica. In: Acidentes por Animais Peçonhentos: Reconhecimento, Clínica e Tratamento (B. Soerensen, org.), pp. 109-138, São Paulo: Editora Atheneu.

CARVALHO, M. A. \& NOGUEIRA, F., 1998. Serpentes da área urbana de Cuiabá, Mato Grosso: Aspectos ecológicos e acidentes ofídicos associados. $\mathrm{Ca}$ dernos de Saúde Pública, 14:753-763.

FONSECA, F., 1949. Animais Peçonhentos. São Paulo:
Empresa Gráfica da Revista dos Tribunais/Instituto Butantan.

GARCIA, F. C. M.; FULINI, D. R.; MENDES, R. P.; BARRAVIERA, B.; MARCONDES-MACHADO, J.; PEREIRA, P. C. M.; SOUZA, L. R.; ZORNOFF, D. C. M. \& MEIRA, D. A., 1994. Estudo clínico-epidemiológico de doentes picados por serpentes venenosas, na Região de Botucatu. Jornal Brasileiro de Medicina, 67:224-232.

GOMES, T.; LOPES, P. \& STAVOLA, M., 1997. Acidentes por Animais Peçonhentos - 1990/1996. Rio de Janeiro: Assessoria de Doenças Imunopreviníveis, Superintendência de Saúde Coletiva, Secretaria de Estado de Saúde do Rio de Janeiro. (mimeo.)

KOUYOUMDJIAN, J. A.; POLIZELLI, C.; KOUYOUMDJIAN, N. C. V.; BELLUOMINI, H. E. \& GISONDI, M., 1990. Acidentes de trabalho ocasionados por serpentes, na região de São José do Rio Preto, Estado de São Paulo, no período de outubro de 1981 a novembro de 1987. Estudo retrospectivo. $R e$ vista Brasileira de Saúde Ocupacional, 18:62-70.

LEBRÃO, M. L.; RIBEIRO, L. A. \& JORGE, M. T., 1995. Avaliação dos óbitos por acidentes por serpentes peçonhentas no Estado de São Paulo, 1988/1989. Revista da Associação Médica Brasileira, 41:343347.

MAGALHÃES, O., 1958. Campanha antiofídica em Minas Gerais. Memórias do Instituto Oswaldo Cruz, 56:291-371.

MARTINEZ, E. G.; VILANOVA, M. C. T.; JORGE, M. T. \& RIBEIRO, L. A., 1995. Aspectos epidemiológicos do acidente ofídico no Vale do Ribeira, São Paulo, 1985 a 1989. Cadernos de Saúde Pública, 11:511515.

MS (Ministério da Saúde)/FUNASA (Fundação Nacional de Saúde), 1998. Manual de Diagnóstico e Tratamento de Acidentes por Animais Peçonhentos. Brasília: MS/FUNASA.

MS (Ministério da Saúde)/FUNASA (Fundação Nacional de Saúde), 2001. Manual de Diagnóstico e Tratamento de Acidentes por Animais Peçonhentos. Brasília: MS/FUNASA.

MS (Ministério da Saúde)/FUNASA (Fundação Nacional de Saúde)/CENEPI (Centro Nacional de Epidemiologia), 1999. Guia de Vigilância Epidemiológica. 4a Ed. Brasília: MS/FUNASA/CENEPI

MS (Ministério da Saúde)/SNABS (Secretaria Nacional de Ações Básicas de Saúde), 1989. Acidentes Ofídicos: Contribuição ao Estudo da Morbidade. Brasília: MS/SNABS. (mimeo.)

NASCIMENTO, S. P., 2000. Aspectos epidemiológicos dos acidentes ofídicos ocorridos no Estado de Roraima, Brasil, entre 1992 e 1998. Cadernos de Saúde Pública, 16:271-276.

PENTEADO, D. C., 1918. Accidentes ophidicos: Effeitos do tratamento específico sobre a mortalidade ophidica. In: Coletânea dos Trabalhos do Instituto Butantan 1901-1917 (Instituto Butantan, org.), pp. 325-331, São Paulo: Instituto Butantan.

PEREIRA NETO, A. F., 2000. Formação de cientista: O caso de Vital Brazil (1865/1950). In: Entre Escombros e Alternativas: Ensino Superior na América Latina (B. V. Schmidt, org.), pp. 99-136, Brasília: Editora Universidade de Brasília. 
PEREIRA NETO, A. F. (org.), 2002. Vital Brazil: Obra Científica Completa. Niterói: Instituto Vital Brazil.

QUEIROZ, L. P. \& MORITZ, R. D., 1989. Acidente botrópico em Florianópolis. Arquivos Catarinenses de Medicina, 18:163-166.

RESENDE, C. C.; ARAÚJO, F. A. A. \& SALLENAVE, R. N. U. R., 1989. Análise Epidemiológica dos Acidentes Ofídicos. Brasília: Ministério da Saúde/Secretaria Nacional de Ações Básicas de Saúde. (mimeo.)

RIBEIRO, E. B., 1950. Vital Brazil. Anais Paulistas de Medicina e Cirurgia, 60:337.

RIBEIRO, L. A.; ALBUQUERQUE, M. J.; PIRES-DECAMPOS, V. A. F.; KATZ, G.; TAKAOKA, N. Y.; LEBRÃO, M. L. \& JORGE, M. T., 1998. Óbitos por serpentes peçonhentas no Estado de São Paulo: Avaliação de 43 casos, 1988/93. Revista da Associação Médica Brasileira, 44:312-318.

RIBEIRO, L. A. \& JORGE, M. T., 1989. Alteração do tempo de coagulação sangüínea em pacientes picados por serpente Bothrops jararaca adulta e filhote. Revista do Hospital das Clínicas da Faculdade de Medicina de São Paulo, 44:143-145.

RIBEIRO, L. A. \& JORGE, M. T., 1990. Epidemiologia e quadro clínico dos acidentes por serpentes Bothrops jararaca adultas e filhotes. Revista do Instituto de Medicina Tropical de São Paulo, 32:436442.

RIBEIRO, L. A.; JORGE, M. T. \& IVERSSON, L. B., 1995. Epidemiologia do acidente por serpentes peçonhentas: Estudo de casos atendidos em 1988. Revista de Saúde Pública, 29:380-388.
RIBEIRO, L. A.; PIRES-DE-CAMPOS, V. A. F.; ALBUQUERQUE, M. J. \& TAKAOKA, N. Y., 1993. Acidente ofídico no Estado de São Paulo. Revista da Associação Médica Brasileira, 39:4-7.

RODRIGUES, D. S.; TELES, A. M. S.; MACHADO, M. A. M. L.; VARGENS, M. M. L.; NASCIMENTO, I. M. \& PLANZO, T. M. P., 1988. Ofidismo na Bahia: Um problema de Saúde Pública. Revista da Sociedade Brasileira de Toxicologia, 1:23-26.

ROSENFELD, G., 1972. Animais peçonhentos e tóxicos do Brasil. In: Introdução à Geografia Médica do Brasil (C. S. Lacaz, R. G. Baruzzi \& W. Siqueira Jr., org.), pp. 430-475, São Paulo: Edgard Blücher/ Edusp.

SILVA Jr., M., 1956. O Ofidismo no Brasil. Rio de Janeiro: Ministério da Saúde/Serviço Nacional de Educação Sanitária.

TORRES, J. B. \& CARLOTTO, O. R., 1982. Levantamento dos gêneros de ofídios e espécies de aracnídeos causadores de acidentes na casuística do centro de informação do Rio Grande do Sul. Memórias do Instituto Butantan, 46:207-218.

TUKEY, J. W., 1977. Exploratory Data Analysis. Reading: Addison-Wesley.

VAZ, E., 1950. Vital Brazil. Anais Paulistas de Medicina e Cirurgia, 60:347-366.

VÊNCIO, D., 1988. Estudo do ofidismo em Goiás: Comprometimento da função renal. Revista Goiana de Medicina, 34:95-116.

Recebido em 3 de junho de 2002

Versão final reapresentada em 20 de setembro de 2002 Aprovado em 8 de outubro de 2002 